\title{
Formulation Development and Characterization of Oxcarbazepine Microemulsion for Intranasal Delivery
}

\author{
Mamatha Tirunagari" , Jamal Sameen', Anitha Nandagopal² \\ ${ }^{1}$ Department of Pharmaceutics, Sultan-ul-Uloom College of Pharmacy, Road No: 3, Banjara Hills, Hyderabad - 500034, \\ Telangana State, India. \\ 2 Department of Pharmacology, Sultan-ul-Uloom College of Pharmacy, Road No: 3, Banjara Hills, Hyderabad - 500034 , \\ Telangana State, India.
}

\begin{abstract}
The objective of this study was to develop novel intranasal microemulsion containing oxcarbazepine (OXC) for treatment of epilepsy. Optimized ratio of Tween 80: Polyethylene glycol and isopropyl myristate was selected after developing pseudoternary phase diagrams and microemulsions were prepared. The prepared microemulsions were characterized for drug content, $\mathrm{pH}$, particle size, polydispersity index, zeta potential, conductivity, viscosity and in vitro release. Ex vivo permeation study for selected microemulsion was performed through sheep nasal mucosa. Further pharmacodynamic performance was evaluated in mice by electrically induced seizures. It was found that selected microemulsion was transparent with average globule size of $20.5 \mathrm{~nm}$ and cumulative percentage drug permeated was $95.60 \%$. Pharmacodynamic evaluation of selected formulation also indicated lesser intensity of seizures with low dose in mice in comparison to oral suspension of OXC. OXC intranasal delivery system is an effective alternate therapy for treatment of epilepsy.
\end{abstract}

Keywords: Oxcarbazepine, intranasal, microemulsion

\section{INTRODUCTION}

Several methods have been reported in the literature to enhance the drug penetration across biological membranes ${ }^{1}$. Nasal drug delivery is an alternate route to oral and parenteral route for the drug to reach systemic circulation. As nasal drug delivery shows various benefits in comparison to other forms of drug deliveries.

Nasal cavity is lined by vascularized epithelium which provides larger surface area useful for drug absorption. It has low enzymatic activity in contrast to the di-

*Corresponding author: Mamatha Tirunagari

E-mail address: tmamatha12@gmail.com 
gestive system. It bypasses hepatic first pass metabolism. So there is negligible irritation of gastrointestinal membrane ${ }^{2-3}$. Nasal drug delivery can be preferred over other routes of drug delivery as it is non-invasive, convenient method with better patient compliance, easy and cost effective ${ }^{4-5}$. Nasal drug delivery also offers advantage of transporting the drugs to brain by detouring the blood brain barriers ${ }^{6}$.

Microemulsions (ME) are clear, thermodynamically stable and isotropic mixtures $^{7}$ (Oil, water and surfactant, mostly along with cosurfactants). ME should be kept under proper storage conditions ${ }^{8}$. There are three types of ME microstructures. They are: Micellar (oil in water), inverted micellar (water in oil) and bicontinuous structure. ME is a new approach to sparingly water soluble drugs, in order to enhance their dissolution and improve bioavailability.

Epilepsy is one of the most common and devastating neurological disorders which is estimated to have a worldwide prevalence of about $0.5^{-1 \%}{ }^{9}$. There are several antiepileptic drugs currently available to control and suppress seizures. However, despite the ongoing development of new pharmacological therapies, more than $30 \%$ of the patients do not become seizure free mainly due to the pharmacoresistance phenomena ${ }^{10}$.

In order to ameliorate the antiepileptic drug regimen, various strategies of administration have been explored. An intranasal microemulsion is one of the advanced drug delivery option, which can be given orally, topically and through nasal cavity as aerosol ${ }^{11}$.

Intranasal ME will transport the drug from nose to brain at a very faster rate. By improved distribution and dissolution of drug within the brain, one can assume reaching higher levels of therapeutic index along with the benefit of reduced side effects, low dosages and also reduction in the cost of therapy ${ }^{12}$.

Intranasal microemulsion formulations have been developed for a number of drugs such as Quetiapine fumarate ${ }^{13}$, Fexofenadine ${ }^{14}$, Diazepam, Lorazepam, Alprazolam $^{15}$, Eucalyptus oil ${ }^{16}$, Ibuprofen ${ }^{17}$ and Zolmitriptan ${ }^{18}$.

Oxcarbazepine (OXC) is an anticonvulsant and mood stabilizing drug, used primarily in the treatment of epilepsy and is also used to treat anxiety / mood disorders. It is a derivative of carbamazepine. Chemically it is 10, 11-dihydro10-oxo- 5 H-dibenz (b,f)azepine-5-carboxamide (As shown in Figure 1). It is poorly soluble in water $(308 \mathrm{mg} / \mathrm{L})$ and has a partition coefficient of 1.31 . It belongs to iminostilbene category of antiepileptic's and act on convulsions by post tetanic potentiation of synaptic transmission, also act on neuropathy by sodium channel blockade and calcium channel blockade mechanism and act on bipolar disorder by decreasing abnormal electrical activity in brain ${ }^{19}$. 


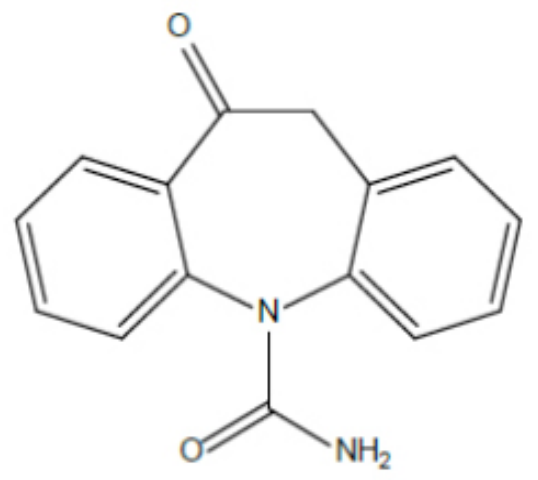

Figure 1: Structure of oxcarbazepine.

The objective of this investigation is to develop intranasal OXC and to compare with oral formulation available in market.

\section{METHODOLOGY}

\section{Materials}

OXC was obtained from Aurobindo Pharma Ltd., Hyderabad, India. Sunflower oil from local market, Isopropyl myristate, Tween 20, Tween 80, PEG 400, PEG 600 , glycerol, propylene glycol, oleic acid, methanol, sodium hydroxide \& potassium dihydrogen phosphate were obtained from S.D. Fine-Chem Ltd., Mumbai. Dialysis membrane was procured from Himedia, Mumbai. All the chemicals were of analytical grade and purchased commercially. Double distilled water was used throughout the study.

Software used: Ternary phase diagram (CHEMIX School 3_60).

\section{Methods}

\section{Solubility Studies}

The solubility of OXC in various components (oils, surfactants and cosurfactants) was determined by adding an excess of drug to $25 \mathrm{ml}$ conical flask, containing 10 $\mathrm{ml}$ of selected vehicle and vortexed for half an hour and placed on a rotary shaker for 48 hours at room temperature. Then contents were centrifuged (REMI R-8C) at 3000 rpm for 5 minutes. The supernatant was filtered through a membrane filter $(0.45 \mu \mathrm{m})$ and the drug concentration in filtrate was determined by UVVisible (UltraViolet) spectroscopy (Thermo Fisher Scientific Model Evolution 201 series). The oil, surfactant and cosurfactant that showed high soloubility of OXC was used in preparation of microemulsions. 


\section{Preparation of Pseudoternary Phase Diagram ${ }^{20}$}

Pseudoternary phase diagrams were constructed to obtain the appropriate ratio of surfactant: cosurfactant which can result in to large existence of microemulsion area. They were constructed using water titration method. Surfactant (Tween 80) and cosurfactant (PEG 600) were mixed (Smix) in different weight ratios (1:1, 2:1, 4:1) and represented as A-series, B-series and C-series respectively. Oil (isopropyl myristate) and Smix (Tween 80 and PEG 600) were mixed thoroughly in different weight ratios from 1:8 to 8:1 in different test tubes and diluted with distilled water in a drop wise manner till it changed from opaque to transparent (composition shown in Table 1). The concentrations of components were recorded in order to complete pseudoternary phase diagrams, and then the contents of oil, surfactant, cosurfactant and water at appropriate weight ratios were selected based on these results.

Table 1: Formulation composition of microemulsions

\begin{tabular}{|c|c|c|}
\hline Formulation code & Smix (Tween 80 and PEG 600) & 0il and Smix \\
\hline A-Series, A1- A8 & $1: 1$ & $1: 8$ to $8: 1$ \\
\hline B-Series, B1 - B8 & $2: 1$ & $1: 8$ to $8: 1$ \\
\hline C-Series, C1 - C8 & $4: 1$ & $1: 8$ to $8: 1$ \\
\hline
\end{tabular}

By joining the change points the boundaries of phases formed were obtained in the phase diagrams. All samples exhibiting a transparent and homogenous state were assigned to a microemulsion area, a monophasic area, in the phase diagram. The pseudoternary phase diagrams were constructed by using CHEMIX School 3_6o software (As shown in Figure 2).

\section{Preparation of Microemulsion}

The OXC microemulsion was prepared by phase titration method employing Isopropyl myristate (IPM) as oil, Tween 80 as surfactant and PEG 600 as cosurfactant.

\section{Preparation of Microemulsion with 1:1, 2:1 And 4:1 Ratio of Smix}

Accurately weighed $50 \mathrm{mg}$ of the drug was added to test tube containing Smix (Tween 80 and PEG 600) in 1:1 ratio. The mixture was shaken on a cyclo mixer until the drug gets properly mixed. Oil (Isopropyl myristate) was then added to the Smix and again shaken for about $10 \mathrm{~min}$. The mixture was diluted with distilled water in a drop wise manner under constant stirring till a transparent microemulsion was achieved.

IPM and Smix were mixed thoroughly in different weight ratios from 1:8 to 8:1 
(A1 to A8). The pseudoternary phase diagrams were constructed to know the area of the microemulsion formed. Similarly microemulsions with 2:1 and 4:1 ratios of Smix and varying ratios of Oil: $\mathrm{Smix}$ (B1 to $\mathrm{B} 8$ and $\mathrm{C} 1$ to $\mathrm{C} 8$ respectively) were prepared and pseudoternary phase diagrams were constructed.

\section{Characterization of Microemulsion}

For the selected three formulations viz., A2, A5 and B3 different characterization tests were done. These three formulations were selected based on transparency, viscosity and amount of water that can be incorporated. C-series of formulations resulted in microemulsions having high viscosity, turbidity and few resulted in gel like consistency after addition of water. These are the reasons for not selecting C-series (Smix 4:1) for further studies.

\section{Drug Content}

The drug content of microemulsion formulation was determined by dissolving 1 $\mathrm{ml}$ of the formulation in $10 \mathrm{ml}$ of methanol. After suitable dilutions with methanol, absorbance was determined using the UV-Visible spectrophotometer keeping blank microemulsion as control at wavelength $256 \mathrm{~nm}$.

\section{pH Determination}

The $\mathrm{pH}$ values of the microemulsions were measured by a $\mathrm{pH}$ meter (Digisun Electronics, India) at ambient temperature with glass electrode.

\section{Particle Size Distribution, Polydispersity Index (PDI), Zeta Potential And Conductivity}

Physical characteristics of microemulsion (particle size distribution, polydispersity index, zeta potential and conductivity) were determined by using Dynamic light scattering (DLS) method using a zetasizer (Horiba SZ-10oZ, Japan).

\section{Viscosity Measurement}

Microemulsions are generally low viscosity systems. The viscosity of prepared microemulsion was measured using Brookfield viscometer (Brookefield viscometer LVDV-E, US).

\section{In vitro Release Studies}

\section{Preparation of Calibration curve of OXC in $40 \%$ v/v PEG 400 plus phosphate buffer pH 6.4}

Accurately weighed $10 \mathrm{mg}$ of drug was dissolved in $100 \mathrm{ml}$ of $40 \% \mathrm{v} / \mathrm{v}$ PEG 400 + phosphate buffer $\mathrm{pH} 6.4$ which gives stock solution of $100 \mu \mathrm{g} / \mathrm{ml}$.

From this stock solution aliquots of $0.5 \mathrm{ml}, 1 \mathrm{ml}, 1.5 \mathrm{ml}, 2 \mathrm{ml}, 2.5 \mathrm{ml}, 3 \mathrm{ml}, 3.5$ $\mathrm{ml}, 4 \mathrm{ml}, 4.5 \mathrm{ml}$ were pipetted out into a series of $10 \mathrm{ml}$ volumetric flasks and 
make up to mark with phosphate buffer which gives $5,10,15,20,25,30,35,40$, $45 \mu \mathrm{g} / \mathrm{ml}$ respectively.

The absorbance of the resulting solution was then measured at $256 \mathrm{~nm}$ using UV spectrophotometer. The calibration curve was obtained by plotting Absorbance vs. Concentration in $\mu \mathrm{g} / \mathrm{ml}$ (Figure 3 ).

\section{In vitro Release Studies}

Based on characterization results three formulations were selected i.e., A2, A5 and $\mathrm{B}_{3}$ for in vitro release studies. The composition of selected formulations is shown in Table 2. The in vitro release study was carried out using Franz diffusion cell (Fabricated locally). The donor compartment was open at the top and was exposed to atmosphere. The dialysis membrane with molecular weight in the range 12,000 to 14,000 (Himedia, Mumbai) was previously soaked for 24 $\mathrm{h}$ in phosphate buffer $\mathrm{pH}$ 6.4. The donor and receptor compartments were held together using a clamp. The receptor compartment contained $13 \mathrm{ml}$ of $40 \% \mathrm{v} / \mathrm{v}$ PEG 400+ phosphate buffer $\mathrm{pH} 6.4$ and stirred with a magnetic capsule operated by a magnetic stirrer (REMI 2MLH, India). The temperature was maintained at $37 \pm 0.5^{\circ} \mathrm{C}$ and the receptor compartment was provided with a sampling port. Samples were collected at preset time points. At each sampling time, $3 \mathrm{ml}$ of sample was removed using a syringe with syringe filter and replaced with fresh $40 \% \mathrm{v} / \mathrm{v}$ PEG 400 in phosphate buffer $\mathrm{pH} 6.4^{21}$.

The concentration of drug was determined using a UV-Visible spectrophotometer at a wavelength of $256 \mathrm{~nm}$. The percentage drug released was calculated and plotted against time (Figure 4).

\section{Ex vivo Permeation Studies}

Two formulations were selected for permeation study based on in vitro release studies. The freshly excised sheep nasal mucosa, except the septum part, was collected from the slaughter house. The membrane was kept in 40\% v/v PEG 400 + phosphate buffer $\mathrm{pH} 6.4$ for 15 min to equilibrate. The superior nasal concha was identified and separated from the nasal membrane ${ }^{22}$. The excised superior nasal membrane was mounted on a Franz diffusion cell ${ }^{23}$. Franz diffusion cell used for ex vivo permeation studies had a diameter of $2 \mathrm{~cm}$ and mucosa of thickness $0.2 \pm 0.01 \mathrm{~mm}$. The receptor compartment was filled with $14 \mathrm{ml}$ of diffusion media. Diffusion media was continuously stirred with a Teflon-coated magnetic bar at a constant rate, in a way that the nasal membrane surface just flushes the diffusion fluid ${ }^{24}$.

Two ml of OXC microemulsion was placed in the donor compartment of Franz diffusion cell. Samples were collected at preset time points and analyzed using 
U.V spectrophotometer. Each sample removed was replaced by an equal volume of diffusion media ( $3 \mathrm{ml})$.

Each study was carried out for a period of $3 \mathrm{~h}$, during which the drug in the receptor compartment $(\mu \mathrm{g} / \mathrm{ml})$ across the sheep nasal membrane was calculated at each sampling point.

The cumulative amount of OXC permeated through mucosa was determined by the following equation:

$$
\mathrm{Q}_{\mathrm{n}}=\mathrm{C}_{\mathrm{n}} \times \mathrm{V}_{\circ}+\sum_{\mathrm{i}=1}^{\mathrm{n}-\mathrm{i}} \mathrm{C}_{\mathrm{i}} \times \mathrm{V}_{\mathrm{i}}
$$

S

Where $\mathrm{C}_{\mathrm{n}}$ is $\mathrm{OXC}$ concentration of receptor medium after each sampling time, $\mathrm{C}_{\mathrm{i}}$ is oxcarbazepine concentration for i sample, $\mathrm{V}_{\circ}$ and $\mathrm{V}$ are the volumes of receiver solution and sample respectively, and $\mathrm{S}$ is the effective diffusion area ${ }^{25}$.

\section{Pharmacodynamic Studies}

Maximal Electroshock: Mice weighing about $25 \mathrm{gm}$ and exhibiting clear hind limb extension phase during electrically induced convulsions were included in the present study. The experimental protocol was approved by Institutional Animal Ethics Committee (IAEC/SUCP/o3/2014). Mice were divided into 3 groups $(n=6)$. The first and second groups were treated orally with OXC suspension (0.5\% Na CMC) and intranasally [23] with OXC microemulsion respectively containing OXC equivalent to $13.5 \mathrm{mg} / \mathrm{kg}$ body weight (using a micropipette attached with low density polyethylene (LDPE) tubing, having $0.1 \mathrm{~mm}$ internal diameter at the delivery site). For intranasal administration, 6o $\mu \mathrm{l}$ (0.2542 mg drug) of microemulsion was instilled equally divided in both the nares of mice. The third group was not subjected to any treatment, served as control. Electroconvulsions were produced by applying current (50 mA, o.2 s) through ear clip electrodes using electroconvulsiometer after 30 min of administration of formulations and different phases of seizures were measured.

Briefly after application of current an immediate severe tonic phase (E phase) was observed which was characterized by maximal extension of the anterior and posterior legs. At the end of tonic phase, clonic phase starts which was characterized by paddling movement of the hind limb and shaking of body. During stupor phase which was observed after tonic and clonic phase mice remained silent without any movement. Recovery time was recorded as total time from starting of tonic phase till animal regains its normal movement (Figure 8). 


\section{RESULTS AND DISCUSSION}

It is estimated that more than $98 \%$ of all small molecules and nearly $100 \%$ of large molecular weight drugs systemically delivered to the central nervous system (CNS), either by oral or intravenous routes, do not readily cross the blood brain barrier and reach the brain parenchyma at pharmacologically active concentrations ${ }^{26}$.

In the light of the current knowledge, drug transport across the nasal mucosa into the CNS depends on a variety of factors that can range from the physicochemical properties of the drug to the formulation design and physiological conditions at the absorption site 27-28. $^{27}$

\section{Solubility Studies}

The solubility of the drug was determined in each component of microemulsion (oils, surfactants and cosurfactants) and was reported. Based on absorbance values (qualitative) oil, surfactant and co surfactant was selected.

Oils - IPM > Oleic acid > Sunflower oil

Surfactants - Tween $80>$ Tween $20>$ Cremophor EL

Co surfactant - PEG 600 > PEG 400 > Isopropyl alcohol > Glycerol > Propylene glycol

Depending on solubility results isopropyl myristate was selected as oil for preparation of microemulsion, tween 80 showed good solubility for $\mathrm{OXC}$ and previous studies have reported improved nasal absorption ${ }^{29}$. Thus tween 80 was selected as surfactant and based on solubility results PEG 600 was selected as cosurfactant. The water solubility of the drug was found to be $308 \mathrm{mg} / \mathrm{L}$.

Microemulsions were prepared by the spontaneous emulsification method (phase titration method) and can be depicted with the help of phase diagrams.

\section{Preparation of Pseudoternary Phase Diagram}

The components that showed maximum solubility were further optimized using pseudoternary phase diagrams as shown in Figure 2. The zone of microemulsion was obtained. 
Smix 1:1

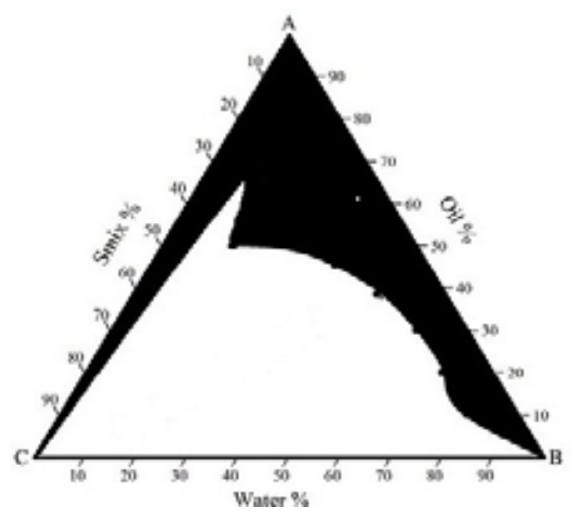

Smix 2:1

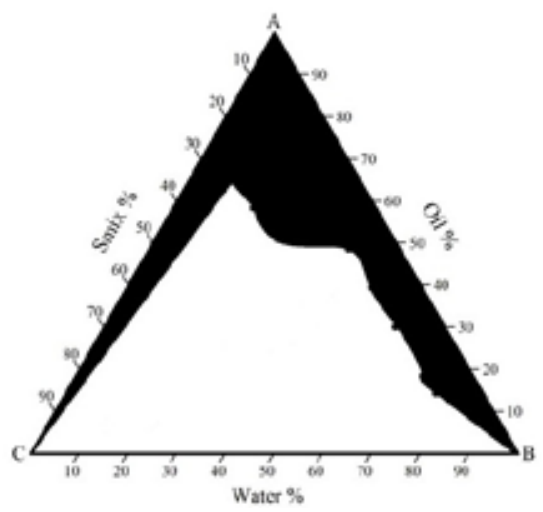

Smix 4:1

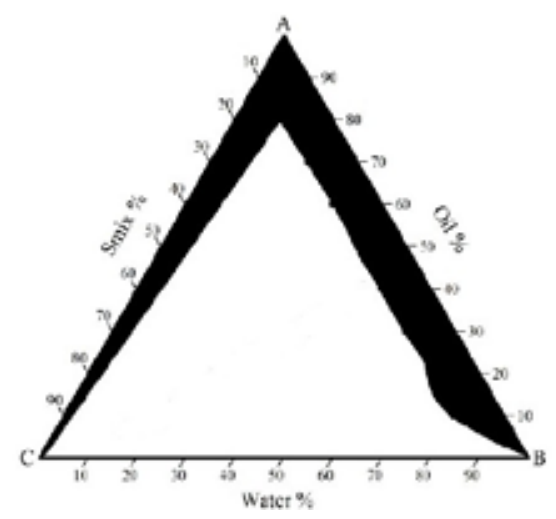

Figure 2: Pseudoternary phase diagram using isopropyl myristate as oil, tween 80 as surfactant, polyethylene glycol 600 as cosurfactant and water (Tween 80: PEG $600=1: 1,2: 1,4: 1$ ).

\section{Preparation of Microemulsion}

Eight formulations (1:8 to 8:1 ratio) from each ratio of Smix (1:1, 2:1 and 4:1) were prepared and the selected formulations were characterized thoroughly. Highest microemulsion area was obtained with ratio of 1:1, 2:1 and thus selected for further studies.

The OXC loaded microemulsion formulations were prepared as per the compositions shown in Table 2. Microemulsion systems were obtained by mixing oil, surfactant and cosurfactant together and adding appropriate quantity of OXC and adding precisely distilled water drop by drop to these oily phases with continuous stirring at ambient temperature. The final concentration of OXC in microemulsion systems was $5 \mathrm{mg} / \mathrm{ml}$. Microemulsions have several specific physic- 
ochemical properties such as transparency, optical isotropy, low viscosity ${ }^{30}$. The formulations having these specific physicochemical properties were selected for characterization, in vitro release and ex vivo permeation studies.

Table 2: Formulation composition of selected microemulsions.

\begin{tabular}{|c|c|c|c|c|}
\hline Formulation code & OXC (mg) & Smix (\%) & IPM (\%) & Water (\%) \\
\hline A2 & 50 & 50 & 14.28 & 35.72 \\
\hline A5 & 50 & 38.46 & 48.07 & 13.47 \\
\hline B3 & 50 & 50.84 & 25.42 & 23.74 \\
\hline
\end{tabular}

\section{Characterization of Microemulsion}

Drug content percentage of all the three selected formulations was found to be $99.25 \pm 1.30$. The $\mathrm{pH}$ was found to be $5.47 \pm 0.42$. Viscosity of all formulation was found to be $60.53 \pm 5.93$ cps. Physical characteristics of microemulsion (particle size distribution, polydispersity index, zeta potential and conductivity) were shown in Figures 3 \& 4.

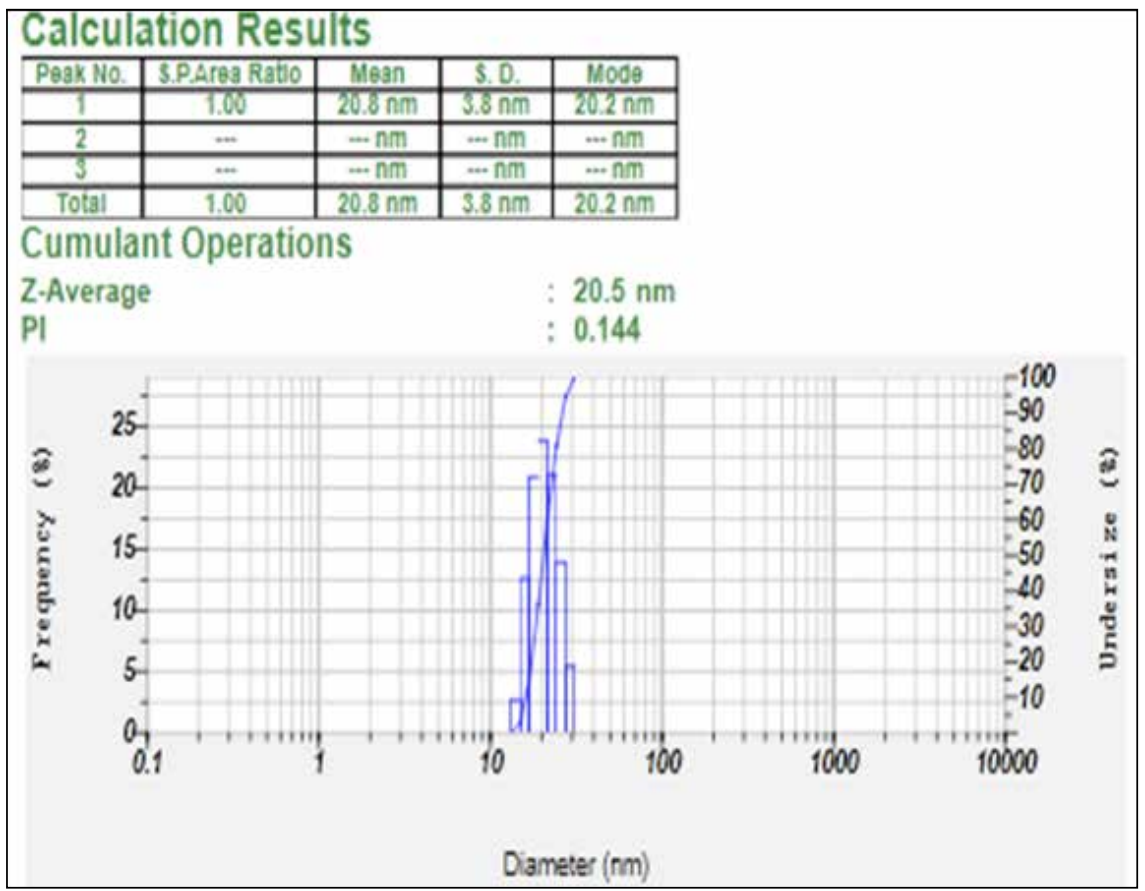

Figure 3: Particle size measurement of Formulation A2. 


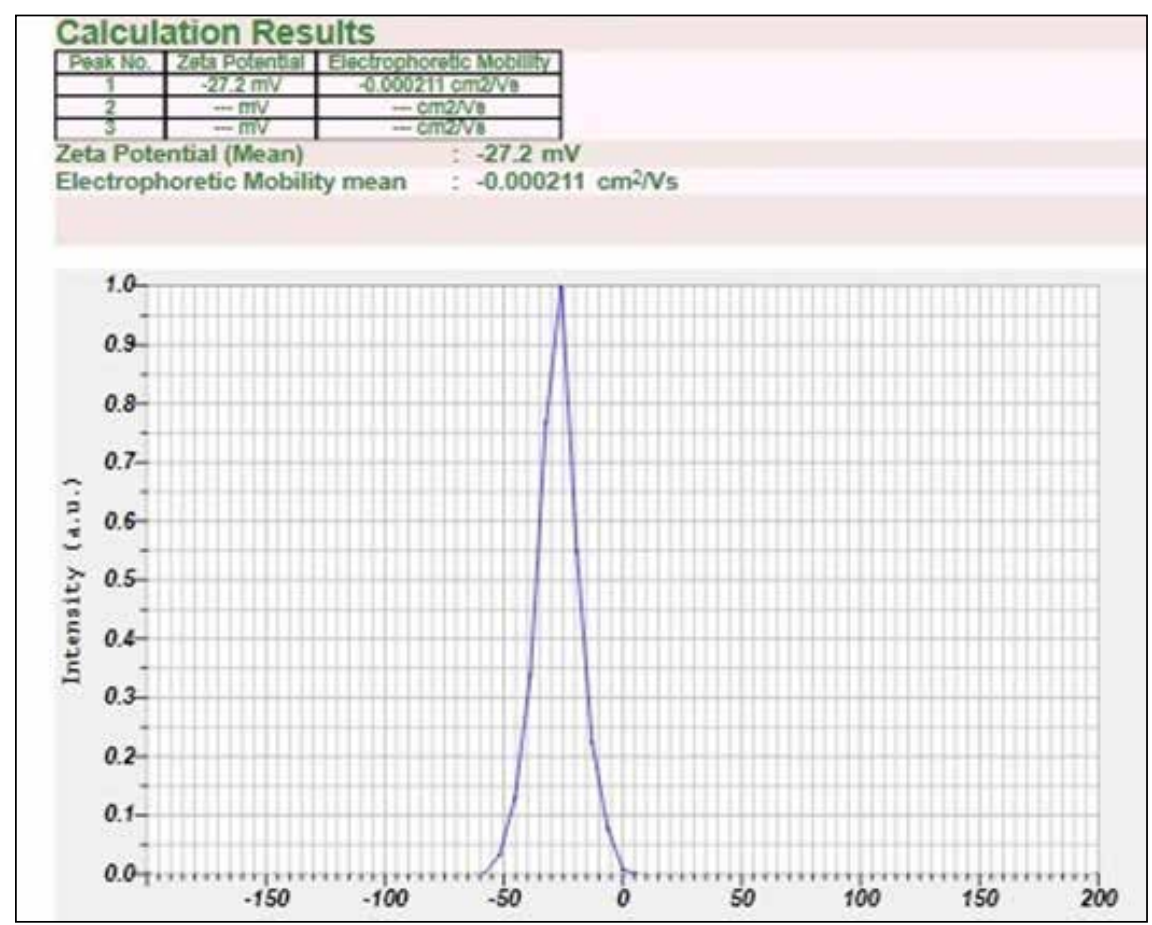

Figure 4: Zeta potential measurement of Formulation A2.

Result of globule size indicated that smallest globule size was obtained with formulation A2 with poly dispersity index 0.144 which is close to zero, indicating that the prepared microemulsion had uniform globule size and thus it was selected for further studies as faster permeation is expected when the globule size is small. Zeta potential was negative which indicated the stability of formulations as there were less chances of globules aggregation. The conductivity of the results confirmed the formation of solution type of microemulsion with water in continuous phase.

\section{Calibration curve of Oxcarbazepine in 40\% v/v PEG $400+$ phosphate buffer pH 6.4:}

Calibration curve of OXC in 40\% v/v PEG 400 + phosphate buffer $\mathrm{pH} 6.4$ was shown in Figure 5 and it was found to be linear. 


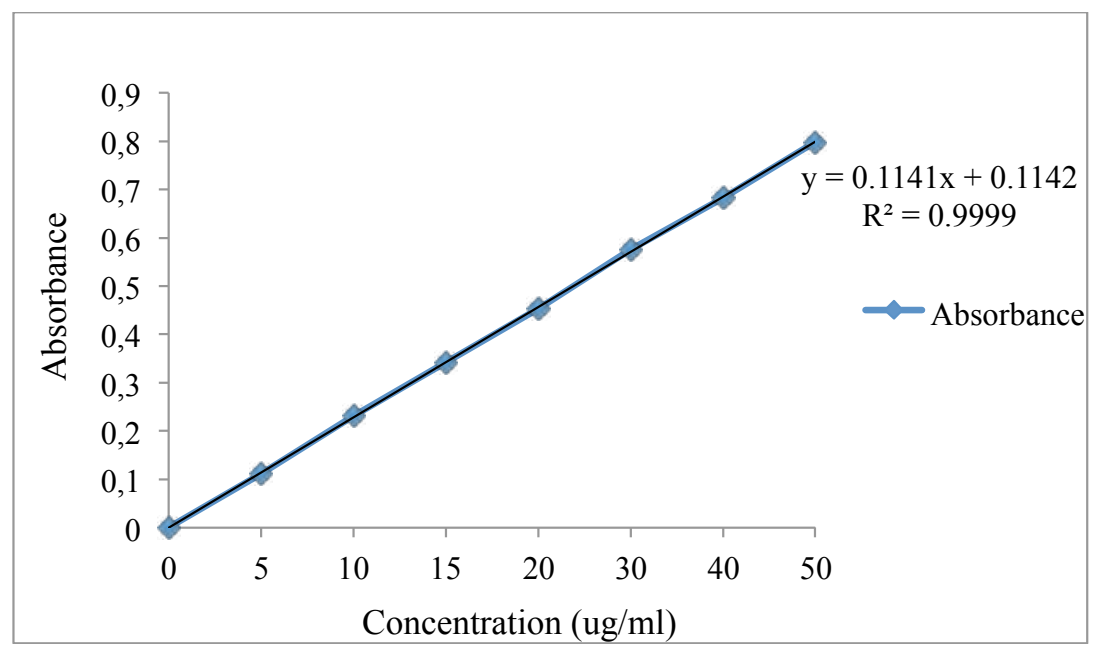

Figure 5: Calibration curve of OXC in 40\% v/v PEG 400 + phosphate buffer pH 6.4

In vitro release studies: The cumulative percentage of drug release after 210 min was found to be maximum with formulation A2 (98.65 \%). In vitro release profile of OXC was shown in Figure 6. The formulation with more amount of water has shown maximum percentage drug release compared to other formulations.

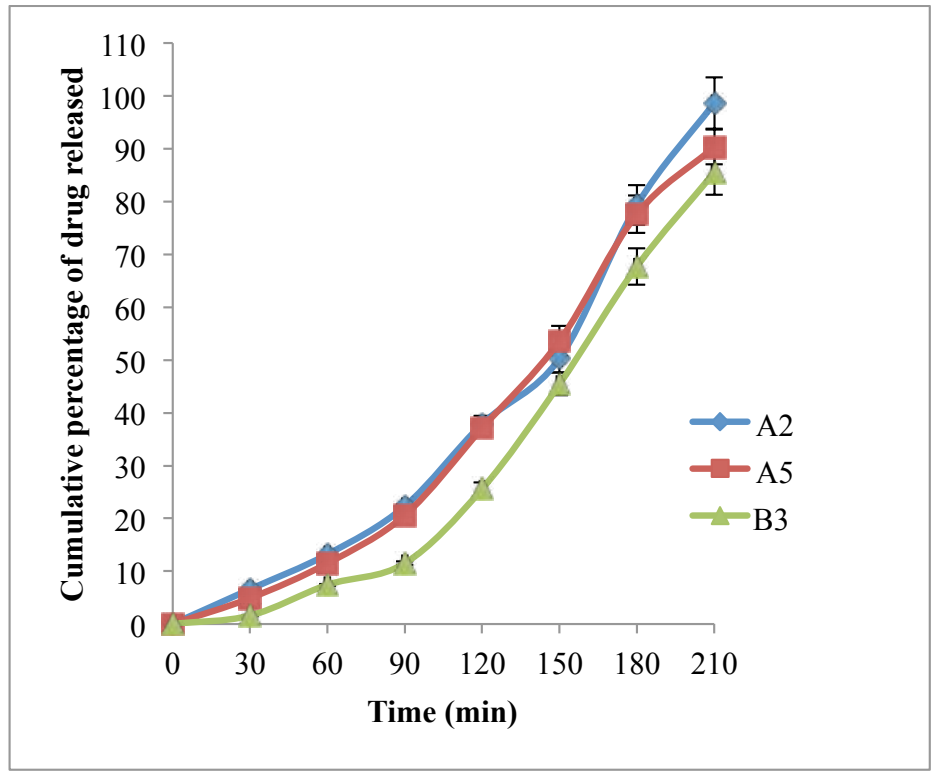

Figure 6: In vitro release profile of OXC. 
Ex vivo permeation studies: Although human nasal mucosa would be the ideal substrate for nasal permeation studies, its limited availability has made to use suitable alternative. It was reported ${ }^{31}$ that the sinus anatomy (including the placement of nasal cavity, turbinates, frontal and maxillary sinuses) in sheep is comparable to humans. Histology of the sheep's nasal mucosa is also identical to that of humans ${ }^{32}$. Hence ex vivo permeation study was performed by using sheep nasal mucosa for optimized formulations A2 and A5. The cumulative percentage of drug permeated after 210 min was found to be maximum with formulation A2 (95.60 \%) and formulation A5 shown 81.25\%. Ex vivo permeation profile of OXC from sheep nasal mucosa was shown in figure 7.

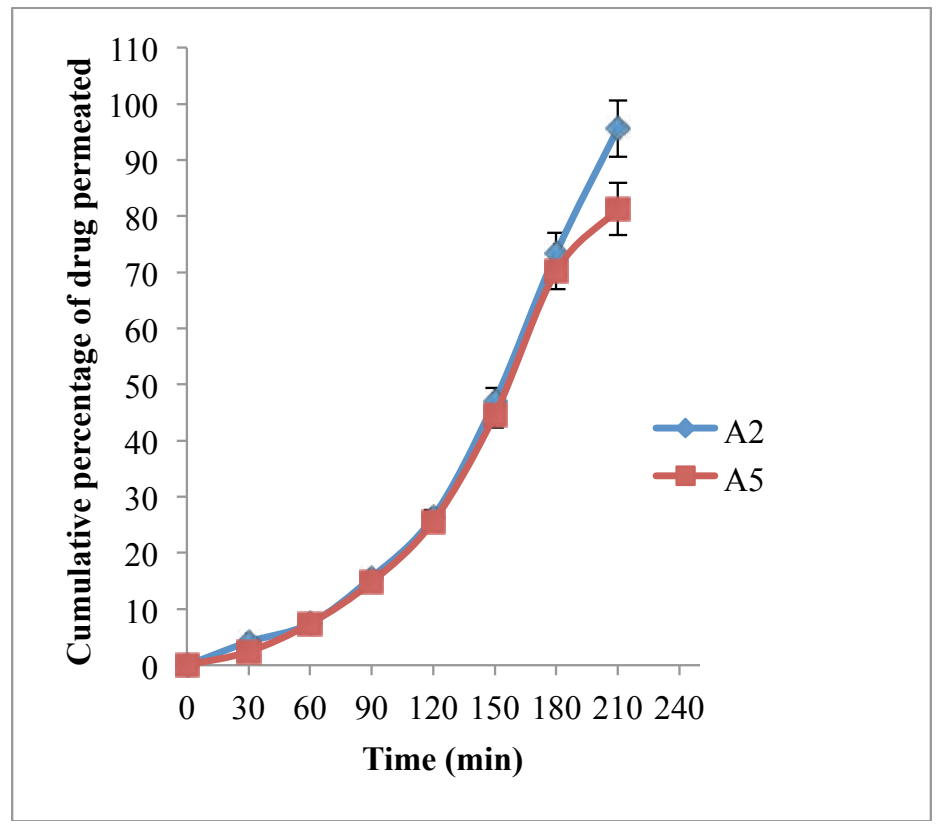

Figure 7: Ex vivo permeation profile of OXC through sheep nasal mucosa.

\section{Pharmacodynamic studies}

The antiepileptic activity was assessed by observing the extent of different phases of seizures including duration of seizures, extension phase, clonus phase and stupor phase and results were represented in Figure 8 and different phases in Figure 9. The results clearly indicated lesser intensity of seizures and rapid recovery from seizures in mice treated with intranasal OXC microemulsion. 


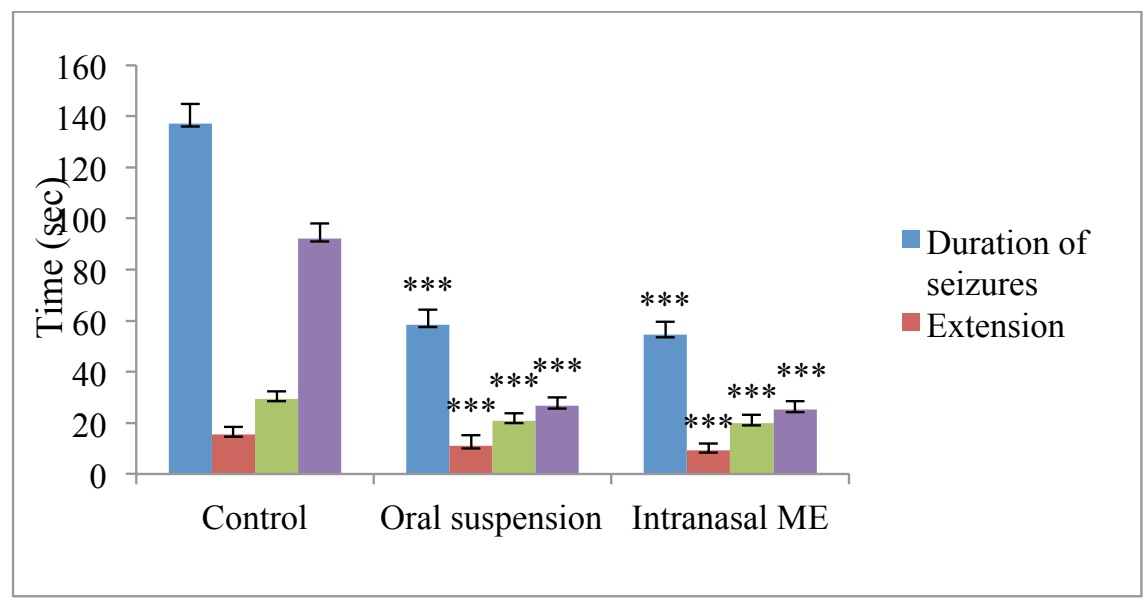

Figure 8: Duration of seizures, extension, clonus and stupor phase for two treatments of oxcarbazepine - OXC oral suspension and OXC microemulsion (IN) where, ${ }^{\star * *}$ indicates significant difference in comparison to control $(p<0.05)$.

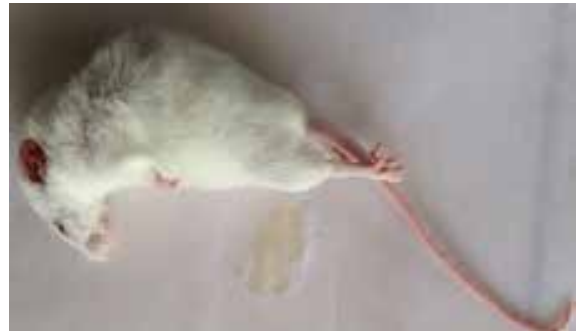

Tonic phase

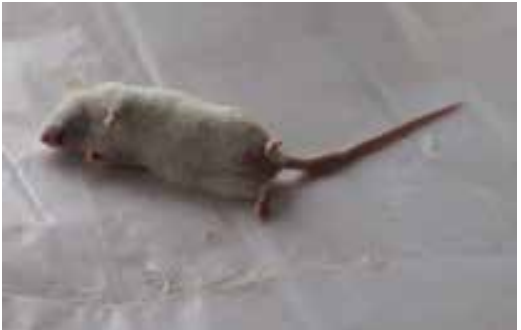

Clonic phase

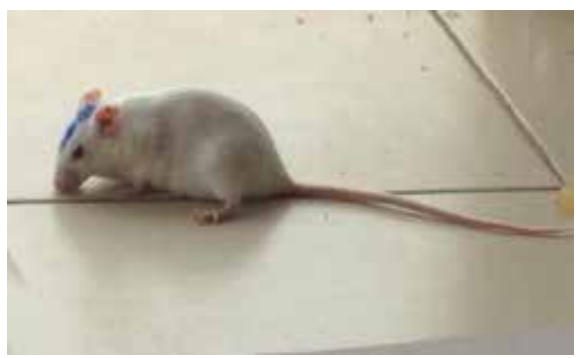

Stupor phase

Figure 9: Different phases of seizures in mice.

The intranasal OXC microemulsion demonstrated lesser intensity of seizures which may be due to larger extent of selective nose to brain delivery of drug in comparison to oral suspension of OXC. This may help in decreasing the dose and frequency of administration of drug and may possibly maximize therapeutic benefits and may also reduce the cost of therapy. 


\section{CONCLUSION}

In comparison to oral formulation, intranasal microemulsion of OXC was shown significant difference in antiepileptic activity. However detailed animal study followed by thorough clinical trials is required to establish clinical safety and efficacy of this formulation.

\section{CONFLICT OF INTEREST}

There is no conflict of interest between the authors of the article.

\section{ACKNOWLEDGEMENT}

We are thankful to our esteemed institution Sultan-Ul-Uloom College of Pharmacy for providing all the necessary facilities to carry out this work.

\section{REFERENCES}

1. Petkar, K.C; Kuchekar, B.S. In-vitro percutaneous absorption of losartan potassium in human skin and prediction of human skin permeability. DARU. 2007, 15(2), 53-60.

2. Eller, N.; Kollenz, C.J.; Bauer, P.; Hitenberger, G. The duration of antidiuretic response of two desmopressin nasal sprays. Int. J. Clinical Pharmacol. Thera. 1998, 36 (9), 494-500.

3. Slot, W.B.; Merkus, F.W.H.M.; Deventer, S.J.H.V.; Tytgat, G.N.J. Normalization of plasma vitamin B12 concentration by intranasal hydroxycobalamin in vitamin B12-deficient patients. Gastroenterology. 1997, 113, 430-433.

4. Ugwoke, M.I.; Norbert, V.; Rennat, K. The biopharmaceutical aspects of nasal mucoadhesive drug delivery. J. Pharm. Pharmacol. 2001, 53, 3-22.

5. Liu, X.F.; Fawcett, J.R.; Thorne. R.G.; Frey, W.H. Intranasal administration of insulin likes growth factor-I by passes the blood-brain barrier and protects against focal cerebral ischemic damage. J. Neurol. Sci. 2001, 87, 91-97.

6. Lisbeth, I. Nasal drug delivery: problems, possibilities solutions. J. Control. Rel. 2003, 87, 187-198.

7. Lawrence, M.J.; Rees, G.D. Microemulsion-based media as novel drug delivery systems. Adv. Drug Deliv. Rev. 2000, 45(1), 89-121.

8. Giustini,Murgia, M.S.; Palazzo, G. Does the Schulman's titration of microemulsions really provide meaningful parameters? Langmuir., 2004, 2O(18), 7381-4.

9. White, H.S. Preclinical development of antiepileptic drugs: past, present, and future directions Epilepsia,2003, 44 (Suppl. 7), 2-8

10. Weaver, D.F. and Pohlmann-Eden, B., (2013) Epilepsia, 54(Suppl. S2), 80-85. Weaver, D.F., Pohlmann-Eden, B., 2013. Pharmacoresistant epilepsy: unmet needs in solving the puzzle(s). Epilepsia, 54(Suppl. S2), 80-85.

11. Kogan, A.; Garti, N. Microemulsions are transdermal drug delivery vehicles. Adv. Colloid Interface Sci. 2006, 123-126, 369-385.

12. Shaji, J.; Poddar, A.; and Iyer, S. Brain-Targeted Nasal Clonazepam Microspheres. Indian J Pharm Sci. 2009, 71(6), 715-718.

13. Patel, N.; Baldaniya, M.; Raval, M.; Sheth, N. Formulation and Development of In Situ Nasal Gelling Systems for Quetiapine Fumarate-Loaded Mucoadhesive Microemulsion. J Pharm Innov. 2015, 10(4), 357-373.

14. Piao, H.M; Balakrishnan, P.; Cho, H.J.; Kim, H.; Kim, Y.S.; Chung, S.J; Shim, C.K; Kim, 
D.D. Preparation and evaluation of fexofenadine microemulsions for intranasal delivery. Int. J.Pharm. 2010, 395(1-2), 309-316.

15. Porecha S.; Shah, T.; Jogani, V.; Naik, S.; Misra, A. Microemulsion based intranasal delivery system for treatment of insomnia. Drug Deliv. 2009, 6(3), 128-34.

16. Tiwari, N. G.; Bajaj, A. N. Formulation development of eucalyptus oil microemulsion for intranasal delivery. Indian J Pharm Sci. 2007, 69 (5), 731-733.

17. Mandal, S.; Mandal, S. D.; Chuttani, K.; Subudhi, B. B. Mucoadhesive microemulsion of ibuprofen: design and evaluation for brain targeting efficiency through intranasal route. Braz. J. Pharm. Sci. 2015, 51(3), 721-731.

18. Vyas, T.K.; Babbar, A.K.; Sharma, R.K.; Misra, A. Intranasal mucoadhesive microemulsions of zolmitriptan: preliminary studies on brain-targeting. J. Drug Target. 2005, 13(5), 317-24.

19. Evelyn Tecoma, S. Oxcarbazepine. International League against Epilepsy. Epilepsia. 1999, $40(5), S_{37-S} 46$.

20. Acharya, S.P.; Pundarikakshudu, K.; Aashish, P.; Anita, L. Preparation and evaluation of transnasal microemulsion of carbamazepine. Asian J Pharm Sci. 2013, 8(1), 64-70.

21. Mamatha, T.; Venkateswara R.J.; Mukkanti, K.; Ramesh, G. Transdermal Therapeutic System of Isradipine: Effect of Hydrophilic and Hydrophobic Matrix on In Vitro and Ex vivo Characteristics. Arch. Pharm. Res. 2010, 33(7), 1025-1033.

22. Cetin, T.; Cansel, K.O.; Ayhan, S.; Yalcin, O.; Umut, T.; Hikmet, A. Nasal absorption of metoclopramide from different Carbopol 981 based formulations: In vitro, ex vivo and in vivo evaluation. Eur. J. Pharm. Biopharm., 2006, 64, 246-254.

23. Samson, G.; Calera, A.G.; Girod, S.D.; Faure, F.; Decullier, E.; Paintaud, G.; Vignault, C.; Scoazec, J.Y.; Pivot, C.; Plauchu, H.; Pirot, F. Ex vivo study of bevacizumab transport through porcine nasal mucosa. Eur. J. Pharm. Biopharm. 2012, 8o, 465-469.

24. Gavini, E.; Rassu, G.; Muzzarelli, C.; Cossu, M.; Giunchedi, P. Spraydried microspheres based on methyl pyrrolidinone chitosan as new carrier for nasal administration of metoclopramide. Eur J Pharm Biopharm. 20o8, 68, 245-52.

25. Hayton, WL.; Chen, T. Correction of perfusate concentration for sample removal. J Pharm Sci 1982, 71, 820-821.

26. Pardridge, W.M. The blood-brain barrier: bottleneck in brain drug development NeuroRx. 2005, 2, 3-14.

27. Pires, A.; Fortuna, A.; Alves, G.; Falcão, A. Intranasal drug delivery: how, why and what for? J. Pharm. Pharm. Sci. 2009, 12, 288-311

28. Vyas, T.K.; Tiwari, S.B.; Amiji, M.M. Formulation and physiological factors influencing CNS delivery upon intranasal administration Crit. Rev. Ther. Drug Carrier Syst. 2oo6, 23, 319-347

29. Hongxia, L.; Matthias, G.; Shengjie, B.; Kyoung, A. K.; Chang-Koo, S.; Chung, S. J.; Kim, D. D. Enhancing effect of surfactants on fexofenadine $\mathrm{HCl}$ transport across the human nasal epithelial cell monolayer. Int $J$ Pharm., 2007, 330, 23-31.

30. Lawrence, M.J.; Rees, G.D. Microemulsion-basedmedia as novel drug delivery systems. Adv. Drug Deliv. Rev. 2000, 45, 89-121.

31. Gardiner, Q.; Oluwole, M.; Tan, L.; White, P.S. An animal model for training in endoscopic nasal and sinus surgery. J. Laryngol. Otol. 1996, 110, 425-428.

32. Illum, L.Nasal drug delivery-recent developments and future prospects. J.Control. Release. 2012, 161, 254-263. 